\title{
Imaging Metal-Organic Frameworks (MOFs) Using Cryo-TEM and Direct Electron-Detection Camera
}

\author{
Li Xing ${ }^{1}$, Huaguang $\mathrm{Yu}^{2 *}$ and Jian-Guo Zheng ${ }^{1 *}$ \\ 1. Irvine Materials Research Institute, University of California, Irvine, CA, USA. \\ 2. College of Physics Science and Technology, Yangzhou University, Yangzhou, P. R. China. \\ * Corresponsing authors: jzheng@uci.edu, hgyu@yzu.edu.cn
}

Metal-organic frameworks (MOFs) are crystalline materials with high porosity and chemical functionality. These porous materials have great potential in various applications, such as catalysis and drug delivery. Recently, we have successfully synthesized two new types of MOFs containing Cd and $\mathrm{Ni}$ elements, respectively. Their crystal structures have been first determined using X-ray diffraction and then confirmed using transmission electron microscopy.

Figure 1 shows the morphology of the Cd-MOFs. Figure 2 displays an annual dark-field (ADF) STEM image of several MOFs crystals (2a) and related elemental maps of $\mathrm{N}(2 \mathrm{~b}), \mathrm{O}(2 \mathrm{c}), \mathrm{Mo}(2 \mathrm{~d}), \mathrm{Cd}(2 \mathrm{e})$ and $\mathrm{P}(2 \mathrm{f})$. There are some challenges to determine the crystal structure of the MOFs using TEM techniques because the MOFs are very sensitive to electron beam radiation. Figure 3 shows a destroyed Cd-MOFs sample which became amorphous as confirmed by the HRTEM image and related FFT pattern (inset).

Using cryo-TEM and Gatan K2 camera, a direct electron detector, we could obtain high quality TEM images under a low dose mode. Figure 4 displays one example of Cd-MOFs lattice image where (020) lattice fringes can be clearly seen. The inset shows the FFT pattern of the image with not only the 020 diffraction spots but also 040 spots.

TEM experiments were carried out on a JEOL 2100F TEM equipped with cryo-EM pole piece and a Gatan 636 cooling holder. The TEM specimen grid was mounted in the holder and cooled down to liquid nitrogen temperature before the gun valve was open. The whole imaging process was conducted at liquid nitrogen temperature with minimal electron dose. Specimen survey was conducted under the diffraction mode, and TEM alignment and image focus were carried out in neighboring carbon area. Overall this setting ensures minimal electron radiation on the area of interest prior to image recording. The images of interest areas were recorded at magnification of $150 \mathrm{kx}$ with the Gatan $\mathrm{K} 2$ camera while the illumination condition was set up to $10 \mathrm{e} / \AA^{2} / \mathrm{s}$. Images were recorded as movies of 20 frames and motion correction was applied to eliminate beam-induced specimen movement. With these imaging conditions, we were able to take 2-dimensional lattice images from both Cd-MOFs and Ni-MOFs to confirm their crystal structures [1].

\section{References:}

[1] The MOFs were synthesized at Yangzhou University and TEM work was conducted at UCI. The authors would acknowledge the use of JEOL 2100F cryo-TEM in IMRI facilities at UCI. 

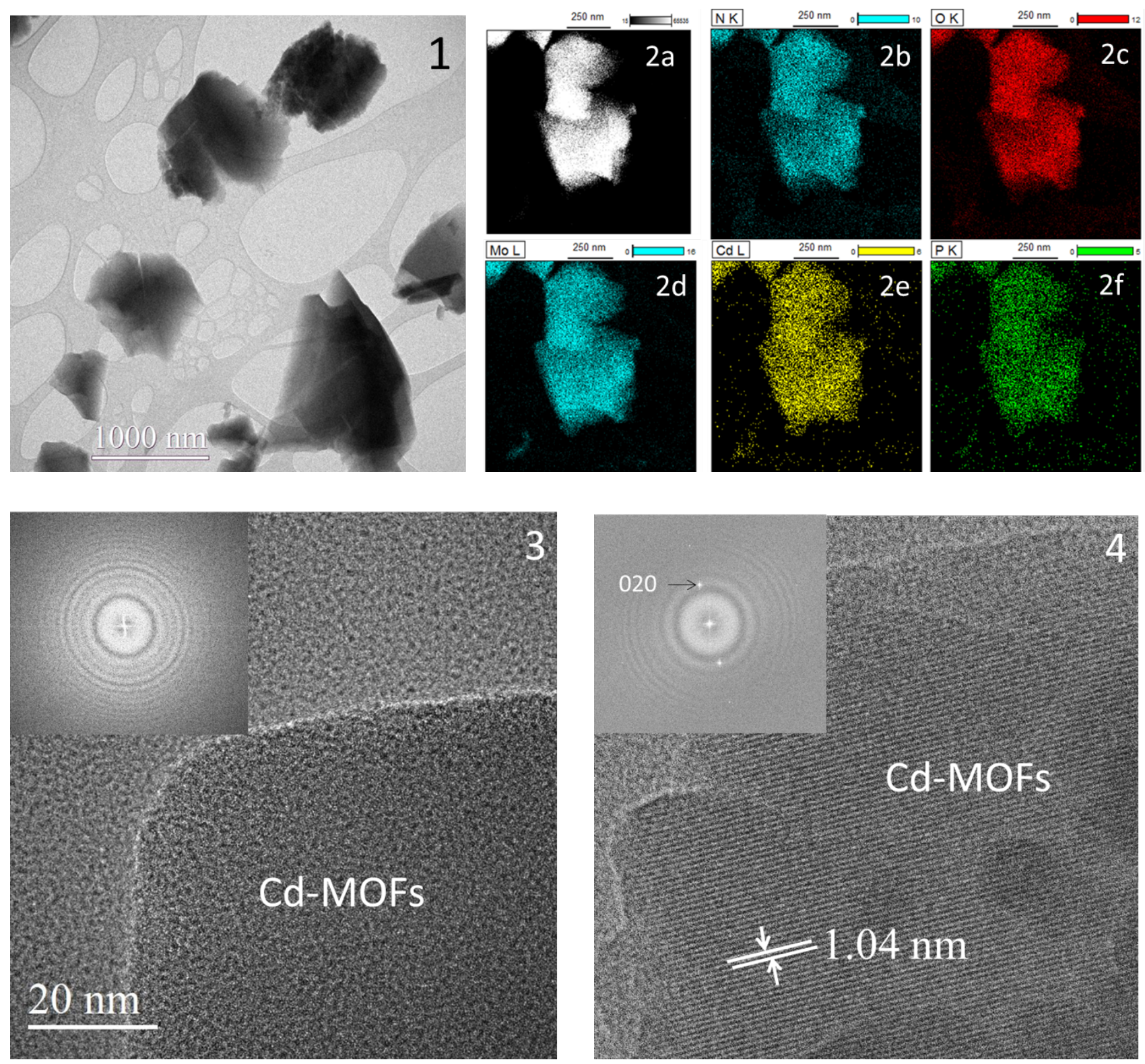

Figure 1. Bright-field TEM image of Cd-MOFs crystals showing sample morphology.

Figure 2. a) ADF STEM image of several Cd-MOFs crystals and their elemental maps containing b) $\mathrm{N}$, c) $\mathrm{O}, \mathrm{d}) \mathrm{Mo}, \mathrm{e}) \mathrm{Cd}$ and f) $\mathrm{P}$.

Figure 3. HRTEM image and its FFT pattern (inset) of a damaged Cd-MOFs sample, displaying an amorphous contrast.

Figure 4. HRTEM lattice image and its FFT pattern (inset) of one Cd-MOFs crystal, showing high quality 020 lattice fringes. 